Int. J. Morphol.,

35(1):172-177, 2017.

\title{
Morphometry of Isan-Thai Clavicles as a Guide for Sex Determination
}

\author{
Morfometría de las Clavículas en una Población Isan-Thai \\ como Guía para la Determinación del Sexo
}

Ailadda Kaewma ${ }^{1}$; Apichakan Sampannang'; Panya Tuamsuk ${ }^{1} \&$ Sitthichai Iamsaard $^{1,2}$

KAEWMA, A.; SAMPANNANG, A; TUAMSUK, P. \& IAMSAARD, I. Morphometry of Isan-Thai clavicles as a guide for sex determination. Int. J. Morphol., 35(1):172-177, 2017.

SUMMARY: The dried clavicles have been well documented to determine individual sex and age in many races. Such morphometric investigations in Isan (Northeastern) Thais have never been reported. Therefore, this study aimed to estimate the sex from dried clavicles of Isan-Thais using morphometric analysis. The identified 454 dried clavicles ( 254 males and 200 females), averaged age (60.69土14.36 years) were measured and analyzed for identification point (IP) and the demarking point (DP) values. The results showed that the identified-bone percentages by IP of the maximum clavicular lengths in males and females were $90.55 \%$ (>139.9 mm) and $89 \%$ ( $<140.6 \mathrm{~mm})$. In addition, such percentages of the mid shaft circumference measured in male clavicles was $83.46 \%(>37.7 \mathrm{~mm})$ and in females was $75 \%(<37.0 \mathrm{~mm})$. However, percentages of the DP in all parameters were less than $50 \%$. The highest DP values of maximum length of male clavicles was $43.31 \%$ (>152.5). In conclusion, the maximum length and mid shaft circumference investigated from this study can be used as basic data of Isan - Thais for applying as a guide in forensic sciences for sex determination from dried clavicle remains.

KEY WORDS: Clavicle; Morphometry; Isan Thais; Sex determination.

\section{INTRODUCTION}

Sex determination from bony remains is important in individual identification. In previous studies, many skeletal human bones have been used to determine and distinguish the sexes, including patella (Dayal \& Bidmos, 2005), sternum (Is, can, 1985), and foot bones (Bidmos \& Dayal, 2003). Particularly, the basic morphometry of clavicles has been reported in many races (Olivier, 1951; Jit \& Singht, 1966; Kaur et al., 2002). In addition, the appearance of rhomboid fossa on inferior clavicular surface could be used to distinguish the males from the females (Jit \& Kaur, 1986; Rogers et al., 2000; Prado et al., 2009). Moreover, the medial articular surface of clavicle could be used to estimate age of Europeans (Kreitner et al., 1998; Falys \& Prangle, 2015). In Thailand, there was only a report investigated about the age estimation based on length measurements by using computed tomography (CT) images of clavicles in North Thais (Pattamapaspong et al., 2015). However, both sex and age estimations including morphometric analysis using CT or dried clavicles in the Isan (Northeastern)-Thai population have never been systemically observed. Therefore, this study aimed to primarily evaluate the morphometry on dried clavicles of Isan Thais using measurement parameters to be hopefully used as a guide for individual sex determination.

\section{MATERIALS AND METHOD}

The 476 identified clavicles (left and right sides) were provided from Isan (Northeastern) Thai skeletons (270 males and 206 females) from KKU Osteological Collection Unit, Department of Anatomy, Faculty of Medicine, Khon Kaen University. The ages were ranged from 19 to 100 years. The incomplete clavicles such as fractured and plate fixation clavicles were excluded. In this study, the lengths of clavicular parameters (Figs. 1 and 2) were measured by digital Vernier caliper in triplicates. These morphometric parameters included $\mathrm{C} 1$ (maximum length), $\mathrm{C} 2$ (mid shaft circumference), C3 (mid shaft maximum diameter), C4 (mid shaft minimum diameter), $\mathrm{C} 5$ (maximum breadth of acromial end) and C6 (maximum breadth of sternal end), respectively (Fig. 1).

\footnotetext{
${ }^{1}$ Department of Anatomy, Faculty of Medicine, Khon Kaen University, 123 Mittaparp Road, Maung, District, Khon Kaen, 40002, Thailand.

${ }^{2}$ Center for Research and Development of Herbal Health Product, Faculty of Pharmaceutical Sciences, Mittaparp Road, Khon Kaen, 40002, Thailand.
} 
In addition, the FL (maximum length of fossa), FW (maximum width of fossa), medial articular surface; ML (maximum length of medial articular surface) and MW (maximum width of medial articular surface), acromial surface; AL (maximum length of acromial surface), and AW (maximum width of acromial surface), respectively, were triplicate measured (Fig. 2). This study was approved from the Office of The Khon Kaen University Ethics Committee for human research (the human ethic number HE581460 to Miss Ailadda Kaewma).

Statistical Analysis. Statistical analyses were performed using the SPSS software version 17.0. The student t-test was used to compare the differences between groups whereas Mann-Whitney U Test was used to analyze the data with non-normal distribution. The p-value $<0.05$ was determined as statistical significance. Identification point (IP) was represented as mean $\pm 1 \mathrm{SD}$ (Udoaka $\&$ Nwokediuko, 2013). To gain more accuracy in sex determinations, the demarking point (DP) based on mean \pm 3 SD differences of all parameters were analyzed (Singh \& Grangrade, 1968).

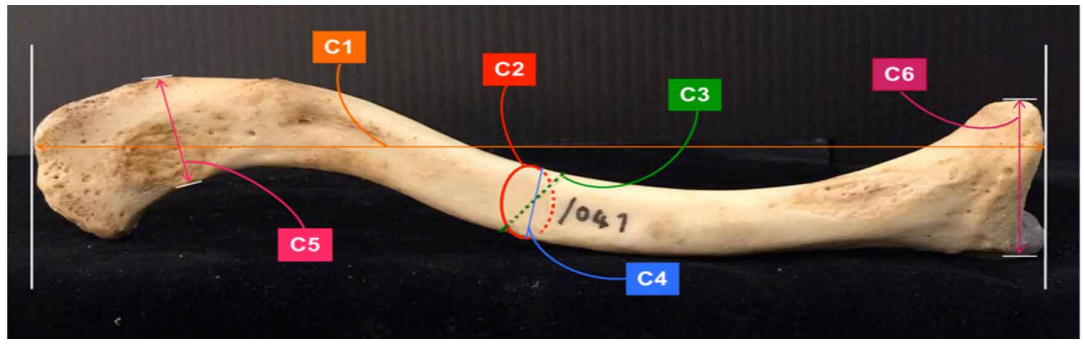

Fig. 1. Photograph showing 6 clavicular parameters measured: $\mathrm{C} 1$ (maximum length), C2 (mid shaft circumference), C3 (mid shaft maximum diameter), C4 (midshaft minimum diameter), C5 (maximum breadth of acromial end) and C6 (maximum breadth of sternal end).
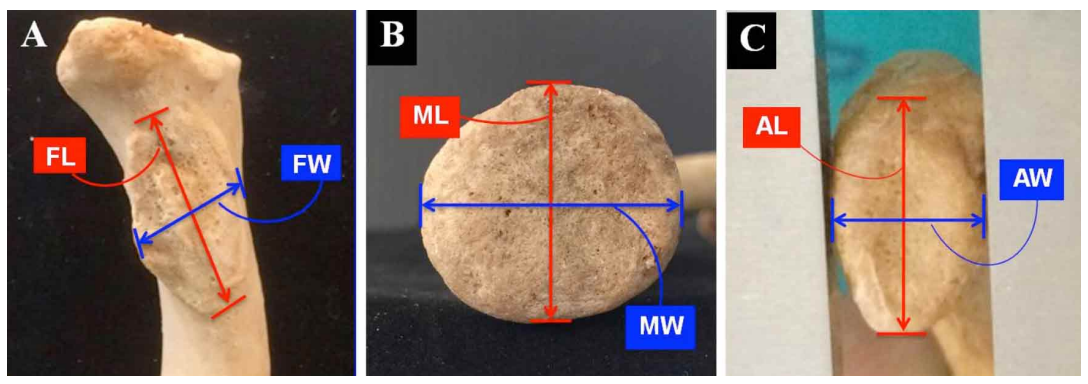

Fig. 2. Photographs showing 6 clavicular parameters measured: A) rhomboid fossa; FL (maximum length of fossa) and FW (maximum width of fossa), B) medial articular surface; ML (maximum length of medial articular surface) and MW (maximum width of medial articular surface), and C) acromial surface; AL (maximum length of acromial surface) and AW (maximum width of acromial surface).

\section{RESULTS}

The results showed that lengths of $\mathrm{C} 1, \mathrm{C} 2, \mathrm{C} 3, \mathrm{C} 4, \mathrm{C} 5$ and $\mathrm{C} 6$ were $141.85 \pm 0.8,37.8 \pm 0.33,10.91 \pm 1.26,10.47 \pm 1.34,21.31 \pm 5.79$ and $23.51 \pm 2.73 \mathrm{~mm}$, respectively. In addition, the lengths of FL, FW, SL, SW,
$\mathrm{AL}$ and AW were $22.82 \pm 4.33,9.81 \pm$ $1.96,23.33 \pm 2.71,23.16 \pm 2.83,17.12$ \pm 3.46 and $11.12 \pm 2.24$, respectively (Table I). Interestingly, all parameters in males were higher than those of females (Table I).

Table II shows the identification point (IP) values; percentage bone identified, and test of significance from 454 Isan Thai clavicles. It was found that the percentage bone identified of the $\mathrm{C} 1$ of both sexes and $\mathrm{C} 2$ of only males is greater than $80 \%$ as compared to the rest parameters (Table II). Significantly, the IPs of $\mathrm{C} 1$ in males and females were $>139.9 \mathrm{~mm}$ and $<140.6 \mathrm{~mm}$. In addition, the IP of $\mathrm{C} 2$ in males was $>37.7 \mathrm{~mm}$ (Table II). The percentage bones identified were composed of $\mathrm{C} 2$ in females $(75 \%$; IP $=<37.0 \mathrm{~mm}), \mathrm{C} 3$ in males $(74.80 \%$; IP $=>10.94)$ and females (76.50\%; IP $=<10.59), \mathrm{C} 4$ in both sexes $(60.24 \%$; IP $=>10.88,57.50$ $\%$; IP $=<9.81), \mathrm{C} 5$ in males $(64.57 \%$; $\mathrm{IP}=>21.88)$, MW in males $(57.87 \%$; $\mathrm{IP}=>24.45)$ and $\mathrm{AL}$ in males $(52.36 \%$; $\mathrm{IP}=>18.36$ ) (Table II). In this study, the percentage bones identified that were lower than $50 \%$ were composed of C5 in females, C6, maximum FL, FW, ML, $\mathrm{SW}$ in females, AL in females and maximum width of AW (Table II). However, all results showed significant differences $(\mathrm{P}<0.001)$ between males and females (Table II).

For further analysis, the demarking point (DP) (mean $\pm 3 \mathrm{SD}$ ) and percentages of identification bone by DP in both sexes were shown in Table III. It was found that no parameter has the percentages of identification bone by DP more than $50 \%$. However, the highest percentage of that of $\mathrm{C} 1$ in males was $43.31 \%(\mathrm{DP}=>152.5 \mathrm{~mm})$ (Table III). As greater than $10 \%$, the percentages of identification bone by DPs of C2, C3, C4 and C5 in males were $25.98 \%(\mathrm{DP}=>43.3), 18.90 \%(\mathrm{DP}=$ $>13.17), 10.24 \%(\mathrm{DP}=>13.31)$ and $10.24 \%(\mathrm{DP}=>27.07)$, respectively (Table III). 
Table I. The lengths of C1 - C6, FL, FW, SL, SW, AL and AW measured in Isan Thais.

\begin{tabular}{|c|c|c|c|c|c|}
\hline $\mathbf{N}$ & Parameters & Sex & Rang (mm) & $\operatorname{Mean}(\mathrm{mm})$ & SD \\
\hline \multirow{3}{*}{1} & \multirow{3}{*}{ Maximum length (C1) } & M & $119-180$ & 150.2 & 0.97 \\
\hline & & $\mathrm{F}$ & $115-152$ & 133.5 & 0.63 \\
\hline & & & Averaged & 141.85 & 0.8 \\
\hline \multirow{3}{*}{2} & \multirow{3}{*}{ Mid shaft circumference (C2) } & M & $31-48$ & 40.7 & 0.37 \\
\hline & & $\mathrm{F}$ & $30-44$ & 34.9 & 0.28 \\
\hline & & & Averaged & 37.8 & 0.33 \\
\hline \multirow{3}{*}{3} & \multirow{3}{*}{ Mid shaft maximum diameter (C3) } & M & $7.89-15.44$ & 11.99 & 1.41 \\
\hline & & $\mathrm{F}$ & $7.74-13.2$ & 9.82 & 1.11 \\
\hline & & & Averaged & 10.91 & 1.26 \\
\hline \multirow{3}{*}{4} & \multirow{3}{*}{ Mid shaft minimum diameter $(\mathrm{C} 4)$} & M & $8.17-16.45$ & 11.26 & 1.46 \\
\hline & & $\mathrm{F}$ & $6.84-14.01$ & 9.67 & 1.22 \\
\hline & & & Averaged & 10.47 & 1.34 \\
\hline \multirow{3}{*}{5} & \multirow{3}{*}{ Maximum breadth of acromial end (C5) } & M & $15.99-31.92$ & 23.32 & 3.20 \\
\hline & & $\mathrm{F}$ & $9.97-27.55$ & 19.29 & 2.59 \\
\hline & & & Averaged & 21.31 & 5.79 \\
\hline \multirow{3}{*}{6} & \multirow{3}{*}{ Maximum breadth of sternal end (C6) } & M & $17.48-33.7$ & 24.38 & 2.88 \\
\hline & & $\mathrm{F}$ & $16.02-28.86$ & 22.64 & 2.57 \\
\hline & & & Averaged & 23.51 & 2.73 \\
\hline \multirow{3}{*}{7} & \multirow{3}{*}{ Maximum length of fossa (FL) } & M & $13.31-32.7$ & 23.46 & 4.32 \\
\hline & & $\mathrm{F}$ & $10.58-33.19$ & 22.18 & 4.34 \\
\hline & & & Averaged & 22.82 & 4.33 \\
\hline \multirow{3}{*}{8} & \multirow{3}{*}{ Maximum width of fossa (FW) } & M & $4.43-16.89$ & 10.39 & 2.28 \\
\hline & & $\mathrm{F}$ & $4.6-14.08$ & 9.22 & 1.63 \\
\hline & & & Averaged & 9.81 & 1.96 \\
\hline \multirow{3}{*}{9} & \multirow{3}{*}{ Maximum length of medial articular surface (ML) } & M & $17.98-34.29$ & 24.21 & 2.89 \\
\hline & & $\mathrm{F}$ & $16.34-28.9$ & 22.45 & 2.52 \\
\hline & & & Averaged & 23.33 & 2.71 \\
\hline \multirow{3}{*}{10} & \multirow{3}{*}{ Maximum width of medial articu lar surface (MW) } & M & $16.25-35.38$ & 24.29 & 3.22 \\
\hline & & $\mathrm{F}$ & $16.67-31.22$ & 22.02 & 2.44 \\
\hline & & & Averaged & 23.16 & 2.83 \\
\hline \multirow{3}{*}{11} & \multirow{3}{*}{ Maximum length of acromial surface (AL) } & M & $9.7-36.15$ & 18.76 & 4.04 \\
\hline & & $\mathrm{F}$ & $9.05-25.35$ & 15.48 & 2.88 \\
\hline & & & Averaged & 17.12 & 3.46 \\
\hline \multirow{3}{*}{12} & \multirow{3}{*}{ Maximum wid th of acromial surface (AW) } & M & $5.74-18.66$ & 12.05 & 2.57 \\
\hline & & $\mathrm{F}$ & $5.59-16.62$ & 10.18 & 1.91 \\
\hline & & & Averaged & 11.12 & 2.24 \\
\hline
\end{tabular}

\section{DISCUSSION}

The mean lengths of $\mathrm{C} 1$ have been also used for sex determination in many populations including English (Parsons, 1961), French (Olivier), Amritsar-zone Indians (Jit \& Singht), USA Negroes (Singh \& Singh, 1972), North America (McCormick et al., 1991), Northwest Indians (Kaur et al.), North Indians (Padeyappanavr et al., 2009), Gujarat-zone Indians (Patel et al., 2009), Iranians (Akhlaghi et al., 2012), and Greeks (Papaioannou et al., 2012). In Isan Thais, the mean length of $\mathrm{C}$ in males $(150.2 \mathrm{~mm})$ was longer than that of females $(133.5 \mathrm{~mm})$. Compared to others, the $\mathrm{C} 1$ of males in all populations previously reported was longer than that of females (Table IV). The difference in this length between males and females may be causes from genetic variations and direct alterations from growth and sex hormones. It might be resulted from the environmental influences such as nutrition, life style activities and occupational stresses (Charisi et al., 2011). It is possible that the estrogen hormone in females can inhibit bone growths as previously explained (Khosla et al., 2012; Cohen et al., 2014; Polur et al., 2015; Nicks et al., 2016). Therefore, this hormone may also affect the long growth of female clavicles as reported in various populations including Isan Thais (Table IV). 
Table II. The identification point (IP), percentage bone identified and test of significance in both sexes $(\mathrm{N}=454)$.

\begin{tabular}{|c|c|c|c|c|c|c|c|}
\hline $\mathbf{N}$ & Parameters & Sex & Range (mm) & $\begin{array}{c}\text { IP }(\mathbf{m m} ; \\
\text { mean } \pm \text { SD) }\end{array}$ & $\mathbf{N}$ & $\begin{array}{c}\text { \%bone } \\
\text { identified by } \\
\text { IP }\end{array}$ & P Value \\
\hline \multirow{2}{*}{1} & \multirow{2}{*}{ Maximum length $(\mathrm{C} 1)$} & M & $119-180$ & $>139.9$ & 230 & 90.55 & $Z=15.76$ \\
\hline & & $\mathrm{F}$ & $115-152$ & $<140.6$ & 178 & 89.00 & $\mathrm{P}<0.0001$ \\
\hline 2 & Mid shaft circumference (C2) & $\begin{array}{l}\mathrm{M} \\
\mathrm{F}\end{array}$ & $\begin{array}{l}31-48 \\
30-44\end{array}$ & $\begin{array}{l}>37.7 \\
<37.0\end{array}$ & $\begin{array}{l}212 \\
150\end{array}$ & $\begin{array}{l}83.46 \\
75.00\end{array}$ & $\begin{array}{l}Z=14.90 \\
P<0.0001\end{array}$ \\
\hline 3 & Mid shaft maximum diameter (C3) & $\begin{array}{c}M \\
F\end{array}$ & $\begin{array}{l}7.89-15.44 \\
7.74-13.2\end{array}$ & $\begin{array}{l}>10.94 \\
<10.59\end{array}$ & 190 & $\begin{array}{l}74.80 \\
76.50\end{array}$ & $\begin{array}{l}Z=13.99 \\
P<00001\end{array}$ \\
\hline 4 & Mid shaft minimum diameter (C4) & $\begin{array}{l}\mathrm{T} \\
\mathrm{F}\end{array}$ & $\begin{array}{l}8.17-16.45 \\
6.84-14.01\end{array}$ & $\begin{array}{l}>10.88 \\
<9.81\end{array}$ & $\begin{array}{l}153 \\
153\end{array}$ & $\begin{array}{l}60.24 \\
57.50\end{array}$ & $\begin{array}{c}\mathrm{Z}=11.81 \\
\mathrm{P}<0.0001\end{array}$ \\
\hline 5 & Maximum breadth of acromial end (C5) & $\begin{array}{c}\mathrm{M} \\
\mathrm{F}\end{array}$ & $\begin{array}{r}15.99-31.92 \\
9.97-27.55\end{array}$ & $\begin{array}{l}>21.88 \\
<16.69\end{array}$ & $\begin{array}{c}164 \\
27\end{array}$ & $\begin{array}{l}64.57 \\
13.50\end{array}$ & $\begin{array}{l}Z=11.50 \\
P<0.0001\end{array}$ \\
\hline 6 & Maximum breadth of sternal end (C6) & $\begin{array}{l}\mathrm{M} \\
\mathrm{F}\end{array}$ & $\begin{array}{l}17.48-33.7 \\
16.02-28.86\end{array}$ & $\begin{array}{l}>25.21 \\
<21.50\end{array}$ & $\begin{array}{l}84 \\
69\end{array}$ & $\begin{array}{l}33.07 \\
34.50\end{array}$ & $\begin{array}{c}\mathrm{T}=6.36 \\
\mathrm{P}<0.0001\end{array}$ \\
\hline 7 & Maximum length of fossa (FL) & $\begin{array}{l}\mathrm{M} \\
\mathrm{F}\end{array}$ & $\begin{array}{l}13.31-32.7 \\
10.58-33.19\end{array}$ & $\begin{array}{l}>26.52 \\
<19.14\end{array}$ & $\begin{array}{l}96 \\
50\end{array}$ & $\begin{array}{l}37.80 \\
25.00\end{array}$ & $\begin{array}{c}Z=5.57 \\
P<0.0001\end{array}$ \\
\hline 8 & Maximum width of fossa (FW) & $\begin{array}{l}\mathrm{M} \\
\mathrm{F}\end{array}$ & $\begin{array}{r}4.43-16.89 \\
4.6-14.08\end{array}$ & $\begin{array}{l}>10.85 \\
<8.11\end{array}$ & $\begin{array}{c}115 \\
50\end{array}$ & $\begin{array}{l}45.28 \\
19.69\end{array}$ & $\begin{array}{c}\mathrm{T}=6.49 \\
\mathrm{P}<0.0001\end{array}$ \\
\hline 9 & Maximum length of medial articul ar surface (ML) & $\begin{array}{l}\mathrm{M} \\
\mathrm{F}\end{array}$ & $\begin{array}{l}17.98-34.29 \\
16.34-28.9\end{array}$ & $\begin{array}{l}>24.97 \\
<21.32\end{array}$ & $\begin{array}{l}95 \\
65\end{array}$ & $\begin{array}{l}37.40 \\
32.50\end{array}$ & $\begin{array}{c}\mathrm{T}=6.61 \\
\mathrm{P}<0.0001\end{array}$ \\
\hline 10 & Maximum width of medial articular surface (MW) & $\begin{array}{l}\mathrm{M} \\
\mathrm{F}\end{array}$ & $\begin{array}{l}16.25-35.38 \\
16.67-31.22\end{array}$ & $\begin{array}{l}>24.45 \\
<21.07\end{array}$ & $\begin{array}{l}147 \\
73\end{array}$ & $\begin{array}{l}57.87 \\
28.74\end{array}$ & $\begin{array}{c}Z=9.76 \\
P<0.0001\end{array}$ \\
\hline 11 & Maximum length of acromial surface $(\mathrm{AL})$ & $\begin{array}{l}\mathrm{M} \\
\mathrm{F}\end{array}$ & $\begin{array}{r}9.7-36.15 \\
9.05-25.35\end{array}$ & $\begin{array}{l}>18.36 \\
<14.72\end{array}$ & $\begin{array}{c}133 \\
80\end{array}$ & $\begin{array}{l}52.36 \\
40.00\end{array}$ & $\begin{array}{c}Z=8.70 \\
P<0.0001\end{array}$ \\
\hline 12 & Maximum width of acromial surface (AW) & $\begin{array}{l}\mathrm{M} \\
\mathrm{F}\end{array}$ & $\begin{array}{l}5.74-18.66 \\
5.59-16.62\end{array}$ & $\begin{array}{c}>12.09 \\
<9.48\end{array}$ & $\begin{array}{c}120 \\
66\end{array}$ & $\begin{array}{l}47.24 \\
33.00\end{array}$ & $\begin{array}{c}\mathrm{T}=8.66 \\
\mathrm{P}<0.0001\end{array}$ \\
\hline
\end{tabular}

Table III. The demarking point (DP) and percentage bone identified by the demarking point in both sexes (N=454).

\begin{tabular}{|c|c|c|c|c|c|c|}
\hline & Parameters & Sex & $\begin{array}{l}\text { Calculated } \\
\text { mean } \pm 3 S D\end{array}$ & $\begin{array}{l}\text { DP } \quad(\mathrm{mm} ; \\
\text { mean } \pm 3 \mathrm{SD})\end{array}$ & $\mathbf{N}$ & $\begin{array}{c}\text { \%bone identified } \\
\text { by DP }\end{array}$ \\
\hline \multirow{2}{*}{1} & \multirow{2}{*}{ Maximum length $(\mathrm{C} 1)$} & M & $121.2-179.3$ & $>152.5$ & 110 & 43.31 \\
\hline & & $\mathrm{F}$ & $114.6-152.5$ & $<121.2$ & 5 & 2.50 \\
\hline \multirow{2}{*}{2} & \multirow{2}{*}{ Mid shaft circumference (C2) } & M & $29.7-51.8$ & $>43.3$ & 66 & 25.98 \\
\hline & & $\mathrm{F}$ & $26.5-43.3$ & $<29.7$ & 0 & 0 \\
\hline \multirow{2}{*}{3} & \multirow{2}{*}{ Mid shaft maximum diameter (C3) } & M & $7.77-16.21$ & $>13.17$ & 48 & 18.90 \\
\hline & & $\mathrm{F}$ & $6.48-13.17$ & $<7.77$ & 1 & 0.50 \\
\hline \multirow{2}{*}{4} & \multirow{2}{*}{ Mid shaft minimum diameter $(\mathrm{C} 4)$} & M & $6.89-15.64$ & $>13.31$ & 26 & 10.24 \\
\hline & & $\mathrm{F}$ & $6.02-13.31$ & $<6.89$ & 1 & 0.50 \\
\hline \multirow{2}{*}{5} & \multirow{2}{*}{ Maximum breadth of acromial end (C5) } & M & $13.73-32.91$ & $>27.07$ & 26 & 10.24 \\
\hline & & $\mathrm{F}$ & $11.50-27.07$ & $<13.73$ & 3 & 1.50 \\
\hline \multirow{2}{*}{6} & \multirow{2}{*}{ Maximum breadth of sternal end (C6) } & M & $15.75-33.02$ & $>30.35$ & 6 & 2.36 \\
\hline & & $\mathrm{F}$ & $14.93-30.35$ & $<15.75$ & 0 & 0 \\
\hline \multirow{2}{*}{7} & \multirow{2}{*}{ Maximum length of fossa (FL) } & M & $10.50-36.41$ & $>35.19$ & 1 & 0.39 \\
\hline & & $\mathrm{F}$ & $9.16-35.19$ & $<10.50$ & 0 & 0 \\
\hline \multirow{2}{*}{8} & \multirow{2}{*}{ Maximum width of fossa $(\mathrm{FW})$} & M & $3.55-17.22$ & $>14.12$ & 9 & 3.54 \\
\hline & & $\mathrm{F}$ & $4.32-14.12$ & $<3.55$ & 0 & 0 \\
\hline \multirow{2}{*}{9} & \multirow{2}{*}{ Maximum length of medial articular surface (ML) } & M & $15.53-32.89$ & $>30.00$ & 7 & 2.76 \\
\hline & & $\mathrm{F}$ & $15.53-30.00$ & $<15.53$ & 0 & 0 \\
\hline \multirow{2}{*}{10} & \multirow{2}{*}{ Maximum width of medial articular surface (MW) } & M & $14.63-33.95$ & $>29.33$ & 17 & 6.69 \\
\hline & & $\mathrm{F}$ & $14.71-29.33$ & $<14.63$ & 0 & 0 \\
\hline \multirow{2}{*}{11} & \multirow{2}{*}{ Maximum length of acromial surface (AL) } & M & $6.65-30.88$ & $>24.12$ & 16 & 6.30 \\
\hline & & $\mathrm{F}$ & $6.84-24.12$ & $<6.65$ & 0 & 0 \\
\hline \multirow{2}{*}{12} & \multirow{2}{*}{ Maximum width of acromial surface (AW) } & M & $4.33-19.77$ & $>15.90$ & 19 & 7.48 \\
\hline & & $\mathrm{F}$ & $4.46-15.90$ & $<4.33$ & 0 & 0 \\
\hline
\end{tabular}


The percentage of bone identified by IP of $\mathrm{C} 1$ in Isan Thais was more than $80 \%$, suggesting for excellent accuracy to be used for sex determination. We concludes that the length of Northeastern - Thai male $\mathrm{C} 1$ must be $>139.9 \mathrm{~mm}$ while of females must be $<140.6 \mathrm{~mm}$. In contrast to Southern Nigerians (Udoaka \& Nwokediuko, 2013), C1's IP was very low in both sexes (male $38.6 \%$ and females $0 \%$ accuracy) as compared with Isan Thais. Similar to others, (Udoaka \& Nwokediuko, 2013; Patel et al.; Padeyappanavr et al.), this study used the DP analysis to increase the accuracy in sex determination. Although the DP of $\mathrm{C} 1$ of Thais was highest as compared to Southern Nigerians, Gujarat zone Indians, Karnataka Indians (Patel et al.; Padeyappanavr et al.; Udoaka \& Nwokediuko, 2013), its accuracy is still lesser than $50 \%$. In this study, the $\mathrm{C} 2$ was also used as a parameter to determine the sexes. In previous investigations (Table V), the means of $\mathrm{C} 2$ of males in many populations were longer than that of females which was similar to Isan Thais. This difference may be affected from estrogen hormone like $\mathrm{C} 1$ growths as described above.
Compared to others, the accuracy of C2'IP to determine males was $83.46 \%$ (>37.7 mm) and female was $75 \%$ ( $<37 \mathrm{~mm}$ ). However, percentage of DP of C2 in Isan Thais was lowest compared to that of Gujarat zone Indians (Patel et al.) and Karnataka Indians (Padeyappanavr et al.). It seemed that the DP of $\mathrm{C} 2$ is not good parameter to be used in sex determination for Isan Thais. Compared to Greeks (Papaioannou et al.), the C4 and C5 in Isan Thais was higher. In contrast, the $\mathrm{C} 3$ and $\mathrm{C} 6$ of Greek were higher than that of this study. In contrast to Southern Nigerians (Udoaka \& Nwokediuko, 2013), the percentages of C5 and C6's IP were very low in both sexes. The percentage of DP of C5 in this study were higher than that DP investigated in Southern Nigerians (Udoaka \& Nwokediuko). However, the accuracy for using C5 and C6 is still not good enough in sex determinations. For the first investigation, the clavicular parameters including FL, FW, ML, MW, AL, and AW were systemically analyzed for sex determination. Unfortunately, although the percentages of all parameters in Isan -Thais were more than $60 \%$ accuracy, their DPs were lesser than $10 \%$. These results suggest that some lengths of Isan clavicle can be used as parameters for sex determination by using IP instead of DP values. .

Table IV. Comparisons of the mean length of clavicle (C1) in both sexes of Isan Thais to other populations

\begin{tabular}{lllll}
\hline Races & N & $\begin{array}{l}\text { Mean }(\mathbf{m m}) \\
\text { Males }\end{array}$ & Females & Reference \\
\hline English & 183 & 154 & 139 & Parsons, 1961 \\
French & 170 & 155 & 138.70 & Olivier, 1951 \\
USA Negroes & 100 & 157.32 & 140.80 & Singh \& Singh, 1972 \\
India (Amritsar zone) & 236 & 147.59 & 129.80 & Jit \& Singht, 1966 \\
North America & 724 & 159 & 141 & McCormick et al., 1991 \\
Northwest Indians & 1000 & 150.27 & 135.37 & Kaur et al., 2002 \\
India (North Interior Karnataka) & 333 & 142.7 & 127.8 & Padeyappanavr et al., 2009 \\
India (Gujarat zone) & 216 & 142.08 & 126.39 & Patel et al., 2009 \\
Iran & 120 & 147.21 & 130.38 & Akhlaghi et al., 2012 \\
Greek & 147 & 153.9 & 137.0 & Papaioannou et al., 2012 \\
Northeastern (Isan) - Thais & 454 & 150.2 & 133.5 & Kaewma et al. (present study) \\
\hline
\end{tabular}

Table V. Comparisons of the mean circumference of clavicle (C2) in both sexes of Northeastern (Isan)Thais to other populations.

\begin{tabular}{lllll}
\hline \multirow{2}{*}{ Races } & \multirow{2}{*}{$\mathrm{N}$} & \multicolumn{2}{c}{ Mean $(\mathrm{mm})$} & References \\
& & Males & Females & \\
\hline Guatemala & 97 & 34.5 & 29.34 & Frutos, 2002 \\
India (North Interior Karnataka) & 333 & 38.15 & 32.11 & Padeyappanavr et al., 2009 \\
India (Gujarat zone) & 216 & 36.77 & 30.16 & Patel et al., 2009 \\
Iran & 120 & 44.07 & 38.38 & Akhlaghi et al., 2012 \\
Greek & 147 & 38.22 & 32.27 & Papaioannou et al., 2012 \\
Northeastern (Isan)- Thais & 454 & 40.7 & 34.9 & Kaewma et al., (pres ent study) \\
\hline
\end{tabular}


ACKNOWLEDGMENTS. This study was financially supported by the invitation research grant (no. IN59125) from Faculty of Medicine, Khon Kaen University to Miss Ailadda Kaewma and Assistant Professor Dr. Sitthichai Iamsaard

A KAEWMA, A.; SAMPANNANG, A; TUAMSUK, P. \& IAMSAARD, I. Morfometría de las clavículas en una población isan-thai como guía para la determinación del sexo. Int. J. Morphol., 35(1):172-177, 2017.

RESUMEN: Las clavículas secas han sido bien documentadas en muchas razas para determinar el sexo y la edad individuales. Tales investigaciones morfométricas en Isan (Noreste de Tailandia) nunca han sido reportadas. Por lo tanto, este estudio tuvo como objetivo estimar el sexo de clavículas secas de IsaisThais utilizando un análisis morfométrico. Se midieron 454 clavículas secas (254 varones y 200 hembras), con una edad media de $60,69 \pm 14,36$ años, y fueron analizadas para los puntos de identificación (IP) y de demarcación (DP). Los resultados mostraron que los porcentajes de hueso identificados por IP en relación a la longitud clavicular máxima en hombres y mujeres fueron $90,55 \%$ (> 139,9 mm) y $89 \%$ (<140,6 mm), respectivamente. Además, tales porcentajes de circunferencia media del eje, medido en las clavículas de los hombres, fue $83,46 \%$ (> 37,7 mm) y en las de mujeres fue $75 \%$ (<37,0 mm). Sin embargo, los porcentajes de la DP en todos los parámetros fueron menores al $50 \%$. Los valores más altos de DP de longitud máxima de las clavículas de los hombres fue 43,31\% (> 152,5). En conclusión, la longitud máxima y la circunferencia media del eje investigados a partir de este estudio se pueden utilizar como datos básicos en Isan - Thais para aplicar como guía en las ciencias forenses para la determinación del sexo a partir de restos de clavícula secas.

PALABRAS CLAVE: Clavícula; Morfometría; Isan Thai; Determinación del sexo.

\section{REFERENCES}

Akhlaghi, M.; Moradi, B. \& Hajibeygi, M. Sex determination using anthropometric dimensions of the clavicle in Iranian population. $J$. Forensic Leg. Med., 19(7):381-5, 2012.

Bidmos, M. A. \& Dayal, M. R. Sex determination from the talus of South African whites by discriminant function analysis. Am. J. Forensic Med. Pathol., 24(4):322-8, 2013.

Charisi, D.; Eliopoulos, C.; Vanna, V.; Koilias, C. G. \& Manolis, S. K. Sexual dimorphism of the arm bones in a modern greek population. J. Forensic Sci., 56(1):10-8, 2011.

Cohen, S. P.; LaChappelle, A. R.; Walker, B. S. \& Lassiter, C. S. Modulation of estrogen causes disruption of craniofacial chondrogenesis in Danio rerio. Aquat. Toxicol., 152:113-20, 2014.

Dayal, M. R. \& Bidmos, M. A. Discriminating sex in South African blacks using patella dimensions. J. Forensic Sci., 50(6):1294-7, 2005.

Falys, C. G. \& Prangle, D. Estimating age of mature adults from the degeneration of the sternal end of the clavicle. Am. J. Phys. Anthropol., 156(2):203-14, 2015.
Is , can, M. Y. Osteometric analysis of sexual dimorphism in the sternal end of the rib. J. Forensic Sci., 30(4):1090-9, 1985.

Jit, I. \& Kaur, H. Rhomboid fossa in the clavicles of North Indians. Am. J. Phys. Anthropol., 70(1):97-103, 1986.

Jit, I. \& Singth, S. The sexing of the adult clavicles. Indian J. Med. Res., 54(6):551-71, 1966.

Kaur, H.; Harjeet; Sahni, D. \& Jit, I. Length and curves of the clavicle in Northwest Indians. J. Anat. Soc. India, 51(2):199-209, 2002.

Kreitner, K. F.; Schweden, F. J.; Riepert, T.; Nafe, B. \& Thelen, M. Bone age determination based on the study of the medial extremity of the clavicle. Eur. Radiol., 8(7):1116-22, 1998.

McCormick, W. F.; Stewart, J. H. \& Greene, H. Sexing of human clavicles using length and circumference measurements. Am. J. Forensic Med. Pathol., 12(2):175-81, 1991.

Nicks, K. M.; Fujita, K.; Fraser, D.; McGregor, U.; Drake, M. T.; McGeeLawrence, M. E.; Westendorf, J. J.; Monroe, D. G. \& Khosla, S. Deletion of estrogen receptor beta in osteoprogenitor cells increases trabecular but not cortical bone mass in female mice. J. Bone Miner. Res.,31(3):606-14, 2016.

Olivier, G. Anthropologie de la clavicule. III, La clavicule due Francis. Bull. Mem. Soc. Anthropol. Paris, 2(4):121-57, 1951.

Padeyappanavr, K. V.; Kazi, A. K. M.; Bhusreddy, P. S. \& Kulkarni, U. K. Sexual dimorphism in adult human clavicles of North Interior Karnataka region. J. Karnataka Med. Leg. Soc., 18(1):3-14, 2009.

Papaioannou, V. A.; Kranioti, E. F.; Joveneaux, P.; Nathena, D. \& Michalodimitrakis, M. Sexual dimorphism of the scapula and the clavicle in a contemporary Greek population: applications in forensic identification. Forensic Sci. Int., 217(1-3):231.e1-7, 2012.

Parsons, F. G. On the proportions and characteristics of the modern English clavicle. J. Anat., 51(Pt. 1):71-93, 1961.

Patel, J. P.; Shah, R. K.; Merchant, S. P.; Niervan, A. B. \& Shah, G. V. Sexing of the human adult clavicle in Gujarat zone. Gujarat Med. J., 64(2):40-6, 2009.

Pattamapaspong, N.; Madla, C.; Mekjaidee, K. \& Namwongprom, S. Age estimation of a Thai population based on maturation of the medial clavicular epiphysis using computed tomography. Forensic Sci. Int., 246:123.e1-5, 2015.

Polur, I.; Kamiya, Y.; Xu, M.; Cabri, B. S.; Alshabeeb, M.; Wadhwa, S. \& Chen, J. Oestrogen receptor beta mediates decreased occlusal loading induced inhibition of chondrocyte maturation in female mice. Arch. Oral Biol., 60(6):818-24, 2015.

Prado, F. B.; de Mello Santos, L. S.; Caria, P. H.; Kawaguchi, J. T.; Preza, A.; Daruge Jnr., E; da Silva, R. F. \& Daruge, E. Incidence of clavicular rhomboid fossa (impression for costoclavicular ligament) in the Brazilian population: forensic application. J. Forensic Odontostomatol., 27(1):126, 2009.

Rogers, N. L.; Flournoy, L. E. \& McCormick, W. F. The rhomboid fossa of the clavicle as a sex and age estimator. J. Forensic Sci., 45(1):61-7, 2000.

Singh, S. \& Singh, S. P. Identification of sex from the humerus. Indian J. Med. Res., 60(7):1061-6, 1972.

Udoaka, A. I. \& Nwokediuko, A. U. Radiologic evaluation of clavicular morphology in Southern Nigerians. Int. J. Morphol., 31(1):94-9, 2013.

Corresponding author:

Dr. Sitthichai lamsaard

Department of Anatomy

Faculty of Medicine, Khon Kaen University

123 Mitraparp Road, Amphoe Muang

Khon Kaen 40002

THAILAND

Email: sittia@kku.ac.th

Received: $18-08-2016$

Accepted: 22-12-2016 